Vietnam Journal of Mechanics, Vol. 27, No. 2 (2005), pp. 74- 85

\title{
PRINCIPLE OF COMPATIBILITY AND NON-GENERALISED COORDINATES
}

\author{
Dinh VAN PhONG \\ Hanoi University of Technology
}

\begin{abstract}
The contribution is devoted to using so-called non-generalised coordinates for deriving the system of equations of motion. Differently from common techniques for constrained mechanical systems the principle of compatibility is chosen as the tool. This takes the advantage in the possibility for extension to systems with nonideal constraints, however, the special treatment is needed. In the article the author derived the theoretical basis as well as numerical algorithms concerning the combination of nongeneralised coordinates with the principle of compatibility. Some comparisons of the technique under consideration and usual technique using Lagrange multipliers are discussed. Some examples are shown for illustration. The case of contact of moving disc on the surface is given in detail
\end{abstract}

\section{INTRODUCTION}

As known the configuration and the motion of mechanical systems are described by a set of coordinates. Various coordinate systems can be used, therefore the sets of coordinates and equations of motion are different to each other. Commonly we can call them, regardless to the physical meaning, as generalised coordinates and use them for deriving equations of motion.

Since generalised coordinates describe the configuration of mechanical system, each coordinate is related to the mass properties of the system. For each coordinate we dispose of one equation of motion in the form of a differential equation of second order. Therefore, if the system is determined by $n$ generalised coordinates we get the system of $\mathbf{n}$ differential equations. Obviously, if these coordinates are not independent, the constraint equations are added to the existing system of differential equations. The system is called the constrained mechanical system, see e.g. [1], [4], [9], [17], [24], [27] etc.

In engineering applications there exists a class of problems where another set of parameters, so-called non-generalised coordinates, is added to the set of coordinates. These coordinates are auxiliary parameters, which, together with generalised coordinates, describe the geometry or other conditions of the mechanical system.

The example of the use of non-generalised coordinates is a contact problem in multibody dynamics. The position of the contact points can be predicted on line during the dynamic simulation by using the nongeneralised coordinates, in this case also called surface parameters, that describe the geometry of the contact surface, see e.g. [18], [28], etc. No inertia forces are associated with these surface parameters. However, the contact constraints are formulated in terms of the system of generalised coordinates and the surface parameters: two points on the two contact surfaces coincide or the normals to the two surfaces at the point contact are parallel. Another example is the control problem with control parameter in the differential equation of motion. The constraint equations include only generalised coordinates that are defined in advance according to the predefined trajectory, see e.g. [7], [11], [16], etc. 
From these examples we can see the important difference between generalised and non-generalised coordinates. For each generalised coordinate one equation of motion in the form of a differential equation is derived, but no equation of motion is generated for nongeneralised coordinates, since no inertia forces are associated with them. Therefore, the nongeneralised coordinates do not increase the number of differential equations of motion.

In this contribution we will derive the theoretical basis and numerical algorithms for using of nongeneralised coordinates for deriving the system of equations of motion. The principle of compatibility and concerning aspects are considered. This principle has an advantage over other ones in the possibility of application even for the system with non ideal constraints, see e.g. [12], [24], etc. Some examples will be discussed for illustration in the section 4 .

\section{NONGENERALISED COORDINATES IN THE SYSTEM OF EQUATIONS OF MOTION}

Let consider a mechanical system with $n$ generalised coordinates. The number of degrees of freedom of the system can be equal or less than $n$. Suppose that for this mechanical system we have $m$ nongeneralised coordinates. The number of constraints for this system, therefore, is greater than $m$. To emphasise that there are $m$ nongeneralised coordinates, we denote the number of constraints for the system is $(s+m)$, with $s>0$ and $n>s$. Hence the degree of freedom of the system is $(n-s)$.

We will use the principle of compatibility to derive the system of equation of motion for the considered constrained mechanical system. The theoretical basis and numerical algorithm are discussed in e.g. [4], [12], [19], [24], etc. Here the resulted equations are extracted directly and we will use them for deriving the basic system of equations with nongeneralised coordinates.

Since there are $n$ generalised coordinates, $n$ equations of motion can be derived in the form:

$$
\mathbf{A} \ddot{\mathbf{q}}=\mathbf{h}+\mathbf{r}_{q}
$$

where $\mathbf{A}$ is the $(n \times n)$-dimensional matrix of inertia, $\ddot{\mathbf{q}}$ is the $(n \times 1)$-dimensional vector of generalised acceleration, $\mathbf{h}$ is a $(n \times 1)$-vector which absorbs all forces acting on the system, excluding the constraint forces, $\mathbf{r}_{q}$ is the $(n \times 1)$-dimensional vector of constraint forces, corresponding to the generalised coordinates $\mathbf{q}$.

If we denote the vector of nongeneralised coordinates as $\mathbf{p}$ which is a $(m \times 1)$-vector, $(m+s)$ constraints for the system can be written:

$$
\mathbf{g}(\mathbf{q}, \mathbf{p}, t)=0
$$

where $\mathbf{g}$ is an $(m+s)$-dimensional vector.

In total (2.1) and (2.2) give $(n+m+s)$ equations, in which the unknowns are: generalised coordinate $\mathbf{q}$, nongeneralised coordinate $\mathbf{p}$ and generalised reaction force $\mathbf{r}$. Keep in mind that generalised reaction force $\mathbf{r}$ has $(n+m)$-elements, corresponding to $(n+m)$ generalised and nongeneralised coordinates, i.e.: 


$$
\mathbf{r}=\left[\begin{array}{l}
\mathbf{r}_{q} \\
\mathbf{r}_{p}
\end{array}\right]
$$

The number of unknowns is $(2 n+2 m)$ and the system of (2.1) and (2.2) is not closed. Other auxiliary equations should be available for defining uniquely unknowns $\mathbf{q}, \mathbf{p}$ and $\mathbf{r}$.

Obviously, the technique for checking the auxiliary conditions depends on the specific problems and on the properties of constraints. For example, for the class of control problems where the requirements are implied to the control forces, these conditions can be written directly. Similarly, for the contact case with friction the relation between the normal and tangential force could be used. In these case we call them the system with nonideal constraints

In the most case of engineering applications for the class of the workless constraints, i.e. the reaction forces do not perform any virtual works, we can find the auxiliary equation by using the criterion of ideality of constraints. Note that this assumption is implicitly applied for all cases with Lagrange multipliers. The approaches described in [8], [19] etc. can be applied in order to get the auxiliary equations. However, in the considered case the application is more complicated due to existence of $\mathrm{m}$ nongeneralised coordinates.

Using pseudoacceleration and the criterion of ideality of constraints leads to the equation, see [19]:

$$
\mathbf{D}^{T} \cdot \mathbf{r}=\mathbf{0}
$$

Matrix $\mathbf{D}$ in this case is an $(n+m) \times(n-s)$-dimensional matrix which can be interpreted as a matrix of coefficients of generalised accelerations $\ddot{\mathbf{q}}$ and $\ddot{\mathbf{p}}$ in relation with $(n-s)$ pseudoaccelerations.

Since $\mathbf{r}$ has two parts $\mathbf{r}_{\mathbf{p}}$ and $\mathbf{r}_{q}$, we can split $\mathbf{D}$ into submatrices $\mathbf{D}_{q}$ and $\mathbf{D}_{p}$ :

$$
\mathrm{D}=\left[\begin{array}{l}
\mathrm{D}_{\mathrm{q}} \\
\mathrm{D}_{\mathrm{p}}
\end{array}\right]
$$

or

$$
\mathbf{D}^{T}=\left[\mathbf{D}_{q}^{T} \mathbf{D}_{p}^{T_{1}}\right.
$$

where $\mathbf{D}_{q}$ is an $n \times(n-s)$-dimensional matrix and $\mathbf{D}_{p}$ is an $m \times(n-s)$-dimensional matrix. The equation (2.4) can be written in the form:

$$
\mathbf{D}_{q}^{T} \mathbf{r}_{q}+\mathbf{D}_{p}^{T} \mathbf{r}_{p}=\mathbf{0}
$$

Matrix $\mathbf{D}$ can be formulated in the similar way described in [19], using constraint equations in combination with pseudoaccelerations. Therefore:

$$
\mathbf{G} \mathbf{D}=\mathbf{0} .
$$

where $\mathbf{G}$ is the Jacobian matrix of constraint $\mathbf{g}$ from (2.2). Note that the dimension of $\mathbf{G}$ is $(m+s) \times(n+m)$. We also can split the matrix $\mathbf{G}$ into two submatrices:

$$
\mathbf{G}=\left[\mathbf{G}_{q} \mathbf{G}_{p}\right]
$$


where $\mathbf{G}_{q}$ is an $(m+s) \times n$-dimensional matrix. The equation $(2.8)$ can be written in the form:

$$
\left[\mathbf{G}_{q} \mathbf{G}_{p}\right] \cdot\left[\begin{array}{l}
\mathbf{D}_{\mathbf{q}} \\
\mathbf{D}_{\mathbf{p}}
\end{array}\right]=0
$$

Basing on (2.8) or (2.10) the techniques describes in [19], [21] can be used to derived $\mathbf{D}$ or $\mathbf{D}_{q}$ and $\mathbf{D}_{p}$. Then the matrix equation from the condition of ideality (2.4) is available for the mechanical system under consideration.

In the case of the system having only generalised coordinates, the equation of the condition of ideality of constraints creates, together with constraint equations and differential equation of motion, a closed system, which can be solved numerically. In the considered case, equation (2.4) performs $(n-s)$ equations, so in total with (2.1) and (2.2), only

$$
n+(m+s)+(n-s)=2 n+m
$$

equations are available for $2 n+2 m$ unknowns $\mathbf{p}, \mathbf{q}, \mathbf{r}$. We should look for other $\mathrm{m}$ equations in order to close the system.

The answer for this question is simple if we recall that $\mathbf{r}$ has two parts: $\mathbf{r}_{q}$ and $\mathbf{r}_{p}$. The reaction forces $\mathbf{r}_{p}$ correspond to the nongeneralised coordinates $\mathbf{p}$ for which no equations of motion are generated. According to the principle of compatibility it implies that:

$$
\mathbf{r}_{p}=\mathbf{0}
$$

This matrix equations give $m$ algebraic equations and the system of equation is closed. But more interesting is a fact that $\mathbf{r}_{p}$ appears only in (2.7) and (2.11) and not in the equations of motion. Therefore (2.4) and (2.11) give:

$$
\mathbf{D}_{q}^{T} \cdot \mathbf{r}_{q}=\mathbf{0}
$$

and the system of equations $(2.1),(2.2),(2.12)$ performs the mixed system of $(2 n+m)$ differential-algebraic equations for also $(2 n+m)$ unknowns: $\mathbf{r}_{q}, \mathbf{q}, \mathbf{p}$.

The obtained system of differential-algebraic equations can be solved by numerical techniques. This problem is intensively investigated by many researches with specialisation in mathematics, numerical mathematics and mechanics see e.g. [2], [3], [5], [6], [14], [23], [26] etc. It is worth noting that the system of differential-algebraic equations (DAE) disposes quite different properties in comparison with the system of ordinary differential equations (ODE) and requires a special treatment. Among important aspects we can emphasise the problems on index, instability on constraints (drift phenomenon), consistency of initial conditions of unknowns etc.

Since for the case of mechanical systems with ideal constraints the more familiar approach is using Lagrange multipliers, we can convert above results to the equations using these multipliers for comparison.

Let $\lambda$ be the column vector of Lagrange multipliers $\lambda=\left[\lambda_{i}\right], i=1, \ldots, m+s$, where the number of multipliers corresponds to the number of constraints. As shown in [20] 
the relation between the reaction force $\mathbf{r}$ and Lagrange multipliers $\lambda$ in the case of ideal constraints is:

$$
\mathbf{r}=\mathbf{G}^{T} \lambda
$$

According to (2.3) and (2.9) $\mathbf{r}$ and $\mathbf{G}$ are split to the submatrices, (2.13) can be written in the form:

$$
\left[\begin{array}{l}
\mathbf{r}_{q} \\
\mathbf{r}_{p}
\end{array}\right]=\left[\begin{array}{l}
\mathbf{G}_{q}^{T} \\
\mathbf{G}_{p}^{T}
\end{array}\right] \lambda
$$

or

$$
\begin{aligned}
& \mathbf{r}_{q}=\mathbf{G}_{q}^{T} \boldsymbol{\lambda} \\
& \mathbf{r}_{p}=\mathbf{G}_{p}^{T} \boldsymbol{\lambda}
\end{aligned}
$$

Putting $\mathbf{r}_{q}$ and $\mathbf{r}_{p}$ to (2.1) and (2.11) we have:

$$
\begin{aligned}
& \mathbf{A} \ddot{\mathbf{q}}=\mathbf{h}+\mathbf{G}_{q}^{T} \boldsymbol{\lambda} \\
& \mathbf{G}_{p}^{T} \boldsymbol{\lambda}=\mathbf{0}
\end{aligned}
$$

The system of equations $(2.17),(2.18)$ and constraint (2.2) creates a closed system of $(n+2 m+s)$ differential-algebraic equations for $(n+2 m+s)$ unknowns $\mathbf{q}, \mathbf{p}, \boldsymbol{\lambda}$. The system can be solved again by numerical techniques.

It is interesting to compare the number of equations and unknowns. The numbers of equations and unknowns for these two approaches with principle of compatibility and Lagrange multipliers are different again, $(2 n+m)$ in the case of the principle of compatibility and $(n+2 m+s)$ in the case of Lagrange multipliers. This will imply the fact that the approach with Lagrange multipliers could have more equations and unknowns when the number of nongeneralised coordinates increases, i.e. if:

$$
m>n-s
$$

It is unlike the case without nongeneralised coordinates where using the principle of compatibility leads always to the system with more unknowns than the system with Lagrange multipliers.

\section{GENERALISED REACTION FORCES IN A SPECIAL CASE}

Now, we will investigate further the reaction components belonging to the specific constraints, which are added to the system due to the presence of the nongeneralised coordinates. Such particular investigation is possible using technique described in [20]. But, below, we will proceed in another way.

For the sake of simplicity we assume, firstly, that the number of constraints under consideration is equal to the number of nongeneralised coordinates, i.e.:

$$
s=0 \text {. }
$$


Hence the system has $n$ generalised coordinates, $m$ nongeneralised coordinates, $n$ differential equation and $m$ constraints:

$$
\begin{aligned}
& \mathbf{A} \ddot{\mathbf{q}}=\mathbf{h}+\mathbf{r}_{q} \\
& \mathbf{g}=\mathbf{0}
\end{aligned}
$$

As discussed above, with ideal constraints the reaction $\mathbf{r}_{q}$ is associated with the matrix $\mathbf{D}_{q}$ that is generated from the Jacobian matrix $\mathbf{G}$ of the constraints $\mathbf{g}$. We can consider two cases of constraints (3.3):

$$
\begin{aligned}
& \mathbf{g}(\mathbf{q}, t)=\mathbf{0} \\
& \mathbf{g}(\mathbf{q}, \mathbf{p}, t)=\mathbf{0}
\end{aligned}
$$

In the 2. case the Jacobian matrix $\mathbf{G}$ as well as $\mathbf{G}_{p}$ and $\mathbf{G}_{q}$ are assumed to be full rank. Using the technique described in [19], the matrix $\mathbf{D}_{q}$ could be derived and is full rank. For the case under consideration $\mathbf{D}_{q}$ has the dimension $(n \times n)$. The system of (2.12), (3.2) and (3.3) creates the system of $2 n$ equations for $2 n$ unknowns $\mathbf{r}$ and $\mathbf{r}_{q}$. The nonsingularity of $\mathbf{D}_{q}$ implies that:

$$
\mathbf{r}_{q}=\mathbf{0} .
$$

In the first case nongeneralised coordinates $\mathbf{p}$ appear only in the equation of notion (3.2). They could be, for example, the controls and the mechanical system should follow the trajectory (3.4). Clearly, one gets:

$$
\mathbf{G}_{p}=0 .
$$

In this case the condition (2.8) leads only to the matrix $\mathbf{D}$ with $(n-m)$ independent columns. Hence, equation (2.12) gives only $(n-m)$ independent relations. However, even in this case we can write the relation (3.6), since $m$ variables appear in the equation (3.2). We get again the closed system of nonlinear equations.

For both cases, the reaction forces of constraints (3.3) are zero. So the system of $(3.2)$ and (3.3) is reduced to:

$$
\begin{aligned}
& A \ddot{q}=h \\
& g=0
\end{aligned}
$$

which performs the mixed system of $(n+m)$ differential-algebraic equations for $(n+m)$ unknowns $\mathbf{q}$ and $\mathbf{p}$.

This conclusion is important and it could be extended for the case when the system is constrained by more conditions than $m$. It is out of scope of this contribution but we can raise the fact that the system of constraints can be split into two groups. The first group is used to construct differential equations of motion and the equation (3.6) is valid for the components of the reaction forces that are corresponding to the second group of constraints, see [21], [22].

From the point of view of numerical solution, the conclusion is interesting too. The system of equations (3.8) and (3.9) can be solved by using the technique for ordinary 
differential equations (ODE). For each integration step the algebraic equation could be solved by using Newton-Raphson method for nonlinear equations. It means that the implicit numerical procedure for evaluating all values of unknowns can be implemented in the algorithm.

\section{ILLUSTRATING EXAMPLES}

In order to illustrate the theoretical basis we will consider some modelled application with nongeneralised coordinates. The first example is the contact problem of the disc on the surface. In general case the surface of a body is described by two parameters and for the point contact problem of two bodies we have in total four nongeneralised coordinates. For illustrating purpose we examine the simplified planar model of one circular disc with radius $r$ that is moving on the curve ground with the radius $R$. The contact point of two bodies is not known in advance at each time instant and we should determine its position.

For the planar curve each point is determined by one parameter in regard to the body fixed frame. In moving body 2 we use the angle ( that denotes the angle of radius vector $\mathrm{CP}$ with the negative axis $\mathrm{O}_{2} y_{2}(P$ is the contact point, the origin of body fixed frame $\mathrm{O}_{2}$ and the centre of gravity $C$ coincide). In non-moving body 1 this parameter is angle $\varphi$ denoting the angle between the radius vector $O_{1} P$ and the negative axis $O_{1} x_{1}$. Besides of two nongeneralised coordinates $\theta$ and $\varphi$ the configuration of the system is defined by 3 generalised coordinates $x_{c}, y_{c}$ and $\Psi$ that determine the position and orientation of the moving body 2 in the inertia system $O x y$ (Fig. 1): $x_{c}$ and $y_{c}$ are the coordinates of the centre of gravity of body 2 and $\Psi$ is the angle of $x$-axis of the body fixed frame and $x$-axis of the inertia system.

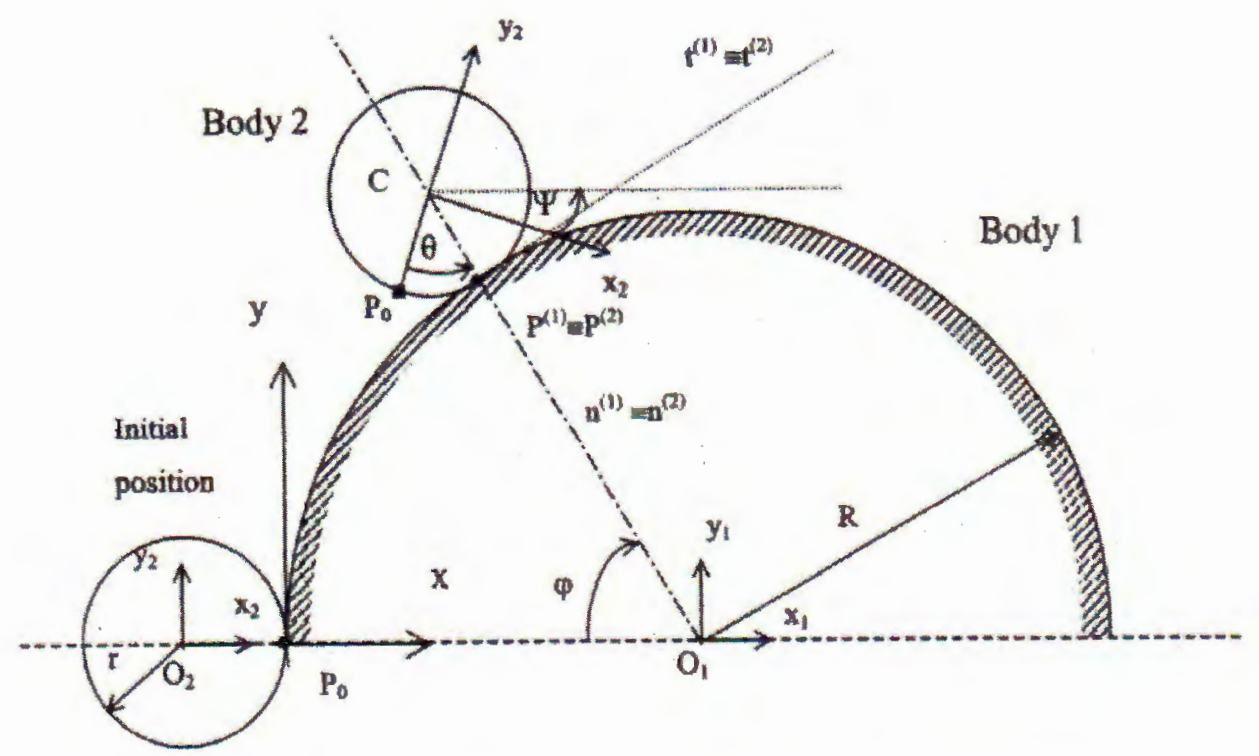

Fig. 1. Contact of rolling disc on the surface 
The system has 2 DOF and we look for 3 constraint equations that are derived from the contact of two bodies 1 and 2. Clearly, in this case $n=3, m=2, s=1$.

Two constraint conditions are derived from the fact that the positions of contact points of two bodies coincide. We can obtain for non-moving body 1 :

$$
\left[\begin{array}{l}
x_{p}^{(1)} \\
y_{p}^{(1)}
\end{array}\right]=\left[\begin{array}{c}
-R \cos \varphi+R \\
R \sin \varphi
\end{array}\right]
$$

The subscript $P$ denotes the coordinates corresponding to the point $P$ and the superscript 1 denotes the body 1 .

For body 2, the body fixed frame moves and we can write:

$$
\left[\begin{array}{l}
x_{p}^{(2)} \\
y_{p}^{(2)}
\end{array}\right]=\left[\begin{array}{cc}
\cos \Psi & \sin \Psi \\
-\sin \Psi & \cos \Psi
\end{array}\right] \cdot\left[\begin{array}{c}
r \sin \theta \\
-r \cos \theta
\end{array}\right]+\left[\begin{array}{l}
x_{c} \\
y_{c}
\end{array}\right]
$$

Hence

$$
\left[\begin{array}{l}
x_{p}^{(2)} \\
y_{p}^{(2)}
\end{array}\right]=\left[\begin{array}{l}
x_{c} \\
y_{c}
\end{array}\right]+r\left[\begin{array}{c}
\sin (\theta-\Psi) \\
-\cos (\theta-\Psi)
\end{array}\right] .
$$

The third equation is derived from the orthogonality of the normal and tangent of the contact surfaces, i.e.

$$
t^{(1)} \cdot n^{(2)}=0
$$

where $t^{(1)}$ is the tangent of the first surface and $n^{(2)}$ is the normal of the second surface. It is easy to calculate the normal $t^{(1)}$ and $n^{(2)}$ :

$$
t^{(1)}=\left[\begin{array}{l}
\sin \varphi \\
\cos \varphi
\end{array}\right]
$$

and

$$
n^{(2)}=\left[\begin{array}{cc}
\cos \Psi & \sin \Psi \\
-\sin \Psi & \cos \Psi
\end{array}\right] \cdot\left[\begin{array}{c}
\sin \varphi \\
\cos \varphi
\end{array}\right]
$$

Hence

$$
n^{(2)}=\left[\begin{array}{c}
\sin (\theta-\Psi) \\
-\cos (\theta-\Psi)
\end{array}\right]
$$

From (4.4), (4.5) and (4.7) we have:

$$
t^{(1)} \cdot n^{(2)}=\sin \varphi \sin (\theta-\Psi)-\cos \varphi \cos (\theta-\Psi)=0
$$

and it yields:

$$
\cos (\theta-\psi+\varphi)=0
$$

Equations of motion can be easily obtained with 3 generalised coordinates. Using the principle of compatibility we have:

$$
\begin{aligned}
& m \ddot{x}_{c}=F_{x}+r_{x} \\
& m \ddot{y}_{c}=F_{y}+r_{y} \\
& J_{c} \ddot{\psi}=M+r_{\psi}
\end{aligned}
$$


where $r_{x}, r_{y}$ and $r_{\psi}$ are generalised reaction forces corresponding generalised coordinates $x_{c}, y_{c}$ and $\psi ; m$ and $J_{c}$ are mass properties of moving body $2 ; F_{x}, F_{y}$ and $M$ are the external forces in the direction of generalised coordinates.

In order to close the system of equation for 8 unknowns $x_{c}, y_{c}, \psi, r_{x}, r_{y}, r_{\psi}, \theta, \varphi$ we have to look for last two equations. They are derived from the condition of ideality of the constraints as described in the theoretical part. Matrix $\mathbf{G}$ in this case is:

$$
\mathbf{G}=\left[\begin{array}{ccccc}
1 & 0 & -r \cos (\theta-\psi) & -R \sin \varphi & \cos (\theta-\psi) \\
0 & 1 & r \sin (\theta-\psi) & -R \cos \varphi & -r \sin (\theta-\psi) \\
0 & 0 & \sin (\theta-\psi+\varphi) & -\sin (\theta-\psi+\varphi) & -\sin (\theta-\psi+\varphi)
\end{array}\right]
$$

From this we get

$$
\mathbf{D}^{T}=\left[\begin{array}{ccccc}
0 & 0 & 1 & 0 & 1 \\
r \cos (\psi-\theta)+R \sin \varphi & r \sin (\psi-\theta)+R \cos \varphi & 1 & 1 & 0
\end{array}\right]
$$

Hence

$$
\begin{aligned}
& r_{\psi}=0 \\
& {[r \cos (\psi-\theta)+R \sin \varphi] r_{x}+[r \sin (\psi-\theta)+R \cos \varphi] r_{y}+r_{\psi}=0}
\end{aligned}
$$

The system of equations (4.3), (4.8)-(4.11), (4.14) and (4.15) is a mixed differentialalgebraic system of 8 equations for 8 unknowns: $x_{c}, y_{c}, \psi, r_{x}, r_{y}, r_{\psi}, \theta, \varphi$. At each timeinstant reaction forces, position and orientation of bodies, as well as the position of contact point can be determined by numerical integration of the considered system of equations of motion,

We can extend the example to the case of rolling without slipping. One more constraint can be applied:

$$
r \theta-R \varphi=0
$$

and the system has only 1 DOF, i.e. $n=3, m=2, s=2$.

Another example is the control problem when the control parameter appears in the equations of motion, see e.g. [13], [25]. The mechanical system is enfurced to follow a predefined trajectory of generalised coordinates that creates a constraint for the system. Therefore in the constraint equations there are only generalised coordinates. The control parameter is the nongeneralised coordinate and could be a parameter of the system or the control force. Again, no inertia forces are associated with these control parameters. Nongeneralised coordinate appears in the differential equations. This is the case we discuss in the section 3 .

\section{CONCLUSION}

In this article we discussed the using of a set of parameters, so-called nongeneralised coordinates, for deriving the system of equation of motion. Since no mass properties of the system are related with these coordinates, in comparison with generalised coordinates, no more differential equations are generated. These parameters are used for describing the 
constraint conditions applied to the system. The conditions may include e.g. the contact constraints or control forces etc.

With different properties, in comparison with the system without nongeneralised coordinates, the system with them needs a specialised treatment when applying the principles of mechanics for deriving the system of equation. Commonly, the method of Lagrange multiplier is usually used, however, the condition of ideality of the constraints is assumed. In this contribution we have dealed with the principle of compatibility which takes the advantage in the possibility for extension for systems with nonideal constraints. The theoretical derivation of equations is evolved in the section 2. As the result we get the system of differential-algebraic equations and the number of equations is in sometime less than the number of equations obtained by the technique with the Lagrange multipliers. This situation never occurs with the generalised coordinates.

The obtained system can be solved only by numerical methods on computers with special techniques for the system of differential-algebraic equations. It is interesting to consider the specialised case when the number of nongeneralised coordinates is equal to the number of constraints. In this case the technique for the system of ordinary differential equations can be used, instead of the algorithm for the system of mixed differentialalgebraic equations that is very useful conclusion from the point of view of numerical solution on computer.

For illustration we discussed some modelled problems with nongeneralised coordinates. The detailed example is devoted to the contact problem when the surface parameters are nongeneralised coordinates. With these parameters the position of the contact point, not known in advance, can be evaluated on line at each time instant with other quantities. The planar model was shown, however, we can apply the algorithm for the three dimensional model.

Acknowledgment. This publication is completed with financial support from the National Basic Research Program in Natural Sciences.

\section{REFERENCES}

1. F. M. L. Amirouche, Computational Methods in Multibody Dynamics, Prentice Hall, Englewood Cliffs, 1992.

2. K. Burrage and L. Petzold, On order reduction for Runge-Kutta methods applied to differential-algebraic systems and to stiff systems of ODE's, SIAM, J.Numer.Anal 27 (1990) 447-456.

3. J. C. Butcher, The Numerical Analysis of Ordinary Differential Equations: Runge-Kutta and General Linear Methods, John Willey \& sons, New York, 1987.

4. V. V. Dobronravov, Osnovy Mechanijky Njegolonomnych System, Vusaja skola, Mockva, 1970.

5. E. Eich-Soellner and C. Fuhrer, Numerical Methods in Multibody Dynamics, B.G.Teubner, Stuttgart, 1998.

6. C. Fuhrer and B. J. Leimkuhler, Numerical solution of differential-algebraic equation for constrained mechanical motion. Numer, Math., 59 (1991) 55-69.

7. A. S. Galiulin, Inverse Problems of Dynamics, Mir Publisher, Moscow, 1984.

8. J. Garcia de Jalon and E. Bayo, Kinematic and Dynamic Simulation of Multibody Systems, The real-time challenge, Springer-Verlag, New York, 1994.

9. H. Goldstein, Classical mechanics, Addison Wesley Publishing Company, Reading, Massachusetts, 1980. 
10. A. Jennings, Matrix Computation for Engineers and scientists, John Willey, 1977.

11. J. N. Juang, M. Q. Phan, Identification and Control of Mechanical Systems, Cambridge University Press, 2001.

12. Nguyen Nhat Le, Dinh Van Phong, Do Sanh, On numerical methods for constrained mechanical systems, Proceedings of IUTAM Symposium on Recent Development in NonLinear Oscillation of Mechanical System (NOMES), Kluwer Academic Publisher, Dodrecht/Boston/London, 1999, 207-216.

13. L. G. Lobas, Niegolonomnjie Modeli Kolesnych Equipazei, Naukova dumka, Kiev, 1986.

14. T. Maly and L. R. Petzold, Numerical methods and software for sensivity analysis of differential-algebraic systems, Applied Numerical Mathematics 20(1996) 57-79.

15. B.W. Morring and J. Genin, Comparison of slip angle and nonholonomic constraint vehicle models, J. Sound and Vibration 65(1979) 85-95.

16. J. I. Nejmark and Fufaev, Dynamics of Nonholonomic Systems, Providence, Ri: American Math. Soc., 1972.

17. P. E. Nikravesh, Computer-aided Analysis of Mechanical Systems, Prentice-Hall, Englewood Cliffs, 1988.

18. F. Pfeiffer and C. Glocker, Multibody Dynamics with Unilateral Contacts, Willey, NewYork, 1996

19. Dinh Van Phong, Principle of compatibility and criteria of ideality in study of constrained mechanical systems, Strojnicky casopis, Journal of Czech and Slovak Mechanical Engineering, Bratislava 47 (1996) 2-11.

20. Dinh Van Phong, An algorithm for calculating reaction forces in constrained mechanical systems, Inzenyrska Mechanika (Engineering Mechanics) 5 (1998) 291-297.

21. Dinh Van Phong, An algorithm for deriving equations of motion of constrained mechanical system, Journal of Mechanics 20 (1999) 1-10.

22. Dinh Van Phong, Successive algorithm for construction of equation of motion of constrained mechanical systems, Vietnam Journal of Mechanics 24 (2002) 101-114.

23. P. J. Rabier and W. C. Rheiboldt, Nonholonomic Motion of Rigid Mechanical Systems from a DAE Viewpoint, SIAM, 2000.

24. Do Sanh, On the Motion of Constrained Mechanical Systems, The thesis of doctor of science, Hanoi University of Technology, Hanoi, 1984.

25. Do Sanh, Dinh Van Phong, Nguyen Nhat Le, Du Quoc Thinh, Investigation of motion of the tricycle along the prescribed trajectory, Proceedings of National Conference on Engineering Vibration, Hanoi, September 5-6, 2000.

26. R. V. Schwerin, Multibody System Simulation, Springer, Berlin/Heidelberg, 1999.

27. A. A. Shabana, Computational Dynamics, John Willey \& sons, New York, 2001.

28. A. A. Shabana, An augmented formulation for mechanical systems with non-generalized coordinates: Application to rigid body contact problems, Nonlinear Dynamics, Kluwer Publisher 24 (2001) 183-204. 


\section{NGUYÊN LÝ PHÙ HợP VÀ CÁC TỌA Độ KHÔNG SUY RỘNG}

Bài báo đề cập đến việc sử dụng các tham số được gọi là các tọa độ không suy rộng trong việc xây dựng các phưong trình chuyển động của các cơ hệ chịu liên kết. Khác với các phương pháp thường được sứ dụng, bài báo đã dùng nguyên lý phù hợp để xây dựng phương trình. Phương án này cho phép mở rộng áp dụng cá với các hệ chịu liên kết không lý tuớng, tuy nhiên các phương trình cơ sờ phải được xứ lý đặc biệt. Tác giả đã xây dựng cơ sớ lý thuyết cũng như các thuật giải liên quan đến việc sử dụng các toạ độ không suy rộng cùng với nguyên lý phù hợp. Một số so sánh giữa phương pháp được xây dựng và phương pháp sư dụng nhân tử Lagrange đã được chì ra. Một số ví dụ được phân tích nhằm mục đích minh họa, trong đó trường hợp tiếp xúc của đĩa chuyển động trên mặt cong được xét một cách chi tiết. 